\title{
Germanica
}

\section{Le médium cinématographique à l'épreuve de la propagande}

L'œuvre américaine de Siegfried Kracauer

Nia PERIVOLAROPOULOU : Das kinematographische Medium im Angesicht der

Propagan-da: Das amerikanische Werk von Siegfried Kracauer

Nia PERIVOLAROPOULOU: The Film Medium Facing Propaganda: Siegfried

Kracauer's American work

\section{Nia Perivolaropoulou}

\section{(2) OpenEdition}

\section{Journals}

Édition électronique

URL : https://journals.openedition.org/germanica/8587

DOI : 10.4000/germanica.8587

ISSN : 2107-0784

Éditeur

Université de Lille

\section{Édition imprimée}

Date de publication : 1 juin 2020

Pagination : 133-148

ISBN : 978-2-913857-45-2

ISSN : 0984-2632

Référence électronique

Nia Perivolaropoulou, « Le médium cinématographique à l'épreuve de la propagande », Germanica [En ligne], 66 | 2ème trimestre 2020, mis en ligne le 02 janvier 2022, consulté le 06 janvier 2022. URL http://journals.openedition.org/germanica/8587 ; DOI : https://doi.org/10.4000/germanica.8587 


\section{Le médium cinématographique à l'épreuve de la propagande.}

\section{L'œuvre américaine de Siegfried Kracauer}

Nia Perivolaropoulou
Paris

Sur le bateau qui au printemps 1941 l'emmène aux États-Unis, Siegfried Kracauer poursuit l'écriture de son manuscrit d'un livre sur le cinéma, commencé à Marseille en 1940, dans lequel résonnent encore ses derniers et intenses échanges avec Walter Benjamin ${ }^{1}$. Ainsi que l'attestent de nombreuses traces, parfois explicites, parfois implicites, disséminées dans ses écrits américains, Kracauer poursuivra cependant unilatéralement leur dialogue jusqu'à son ultime ouvrage, L'Histoire. Des avant-dernières choses $^{2}$. Il lui reviendra dès lors de proposer une théorie qui de facto intègre leurs réflexions sur la dimension spécifiquement documentaire du film

1. - Sur ces échanges voir dans ce même numéro l'article de Philippe Despoix, « Le médium cinématographique à l'épreuve de la propagande. Un dialogue interrompu entre Siegfried Kracauer et Walter Benjamin », supra, p. 117-132.

2. - Un des moments forts de ce « dialogue » est la confrontation avec les thèses «Sur le concept d'histoire », que Kracauer a menée dès les premiers travaux préparatoires, en 1960, au projet d'un essai sur l'histoire. Ainsi qu'il apparaît à la lecture de ses annotations et commentaires, cette confrontation a pu contribuer à l'éclaircissement de sa propre conception du temps historique. Cf. Siegfried Kracauer, L'Histoire. Des avant-dernières choses, [1969], trad. par C. Orsoni, Paris, Stock, 2006, en particulier le chapitre « Ahasver ou l'énigme du temps », ainsi que la postface des éditeurs ; voir aussi Stephanie Baumann, Im Vorraum der Geschichte. Siegfried Kracauers « History. The Last Things Before the Last », Paderborn, Konstanz University Press, 2014, notamment p. 131-132 et 143-144.

GERMANICA, 2020, LXVI, pp. 133 à 148. 
comme sur la fonction de l'image fixe dans une perspective qui leur était commune, à savoir que l'apparition des médias photo-cinématographiques avait modifié la fonction de l'art. C'est là le sens de l'expression kracauerienne : si le cinéma est un art, il est un art with a difference.

Dans les toutes premières pages de l'« Esquisse de Marseille», Kracauer formule sous une forme aporétique une question exprimant l'importance qu'il accordait à la propagande cinématographique pour toute approche théorique du film : «Le puissant effet de propagande du film paraît inconciliable avec son inhérente capacité de dégonflage. Où placer [cela] ? Comment résoudre le conflit ${ }^{3}$ ? ». Kracauer ne répondra pas directement, on s'en doute, à cette question qui résume de manière lapidaire l'enjeu, pour ne pas dire le défi, que représente la propagande pour le médium cinématographique. De facto, la réponse sera donnée par l'élaboration même d'une théorie qui achèvera le dépassement de la critique de l'idéologie par l'établissement d'une puissance critique sui generis et ancrée dans l'esthétique, au sens d'aisthesis, du film.

\section{De l'" Esquisse de Marseille " à Théorie du film}

En 1938, la dernière phrase de l'étude sur la propagande totalitaire proposait une image frappante : " Derrière le tumulte de la propagande totalitaire surgit une tête de mort ${ }^{4} \gg$. Elle s'inscrivait alors dans le contexte d'une discussion sur l'idéologie et la spécificité de la propagande fasciste et national-socialiste, qui lui conférait une signification sans épuiser pour autant son pouvoir d'évocation. Aussi, l'image de la tête de mort réapparaît-elle, transformée, dans l'« Esquisse de Marseille ». Elle relie directement les deux écrits tout en prenant place dans le cadre d'une réflexion plus large, qui oppose tout ce qui est de l'ordre de l'intention, et par conséquent tout discours, idéologique ou pas, à ce qui lui échappe et qui relève de l'ordre de la matérialité :

Le film enchevêtre dans son jeu l'ensemble du monde matériel, il met pour la première fois - en allant au-delà du théâtre et de la peinture l'étant en mouvement. [...] C'est le rebut qui l'intéresse, ce qui est - à l'intérieur comme à l'extérieur de l'homme. Le visage dans le film n'a pas de valeur si la tête de mort qui se trouve derrière n'affleure pas : « Danse macabre ». Mais à quelle fin ? Cela reste à voir ${ }^{5}$.

3. - Sauf mention contraire, nous traduisons. «Der starke Propagandaeffekt des Films scheint mit der ihm gegebenen Fähigkeit zur Dégonflage unvereinbar. Wo unterbringen? Wie den Widerstreit auflösen? », Siegfried Kracauer, [« Marseiller Entwurf » zu einer Theorie des Films] [1940-41], in : Id., Werke, Bd. 3, Frankfurt a. M., Suhrkamp, 2005, p. 524.

4. - Siegfried Kracauer, « Totalitäre Propaganda » [1938], in : Id., Werke, Bd. 2.2, Studien zu Massenmedien und Propaganda, Berlin, Suhrkamp, 2012, p. 173 : « Hinter dem Tumult der totalitären Propaganda taucht ein Totenkopf auf. »

5. - S. Kracauer, [« Marseiller Entwurf » zu einer Theorie des Films], op. cit., 
L'image, dont la signification ne saurait être surestimée, revient encore en 1949 dans un exposé de son projet d'une esthétique du film. Elle donne son titre au dernier chapitre prévu :

\section{La tête de mort}

S'appuyant sur une analyse de Death day, un court métrage constitué à partir de matériaux filmiques tournés au Mexique par Eisenstein, le dernier chapitre ne se contentera pas de récapituler tout le livre, mais il formulera aussi certaines conclusions de grande portée 6 .

Si l'image de la tête de mort disparaît dans Théorie du film, l'« Epilogue » n'en développe pas moins, ainsi qu'il avait été envisagé dans l'exposé de 1949, la portée et la signification de cette théorie. Lors d'une controverse au moment de la préparation de l'édition allemande du livre qui se cristallisait autour du sous-titre et de l'épilogue, Kracauer affirmera à Adorno qu'il lui est impossible de supprimer « ce chapitre vers lequel toute ma théorie du film converge et pour lequel elle a été écrite ${ }^{7} »$. Au centre du dernier des sous-chapitres de l'épilogue, qui donne son soustitre au livre «La rédemption de la réalité matérielle », figure le passage sur « La tête de Méduse », ultime et radicale métamorphose de l'image de la tête de mort, qui déploie toutes les potentialités présentes in nuce dans les versions précédentes tout en allant bien au-delà.

Cependant, Théorie $d u$ film ne paraîtra que vingt années après sa première version, l'« Esquisse de Marseille ». L'écriture du livre, maintes fois interrompue par des impératifs professionnels, avait revêtu, ainsi que l'attestent sa correspondance et les nombreuses strates de travaux préliminaires, une importance quasi-existentielle. Entre temps, Kracauer publiera From Caligari to Hitler (1947) et participera aux travaux sur la propagande aux États-Unis dans les années 1940 et $1950^{8}$. Ces derniers, effectués dans le cadre de la recherche sur la communication de masse,

p. 531 : «Der Film verwickelt die ganze materielle Welt mit ins Spiel, er versetzt zum ersten Mal - über Theater und Malerei hinausgreifend - das Seiende in Umtrieb. [...] Der Abhub interessiert ihn, das, was da ist - am Menschen selber und außerhalb des Menschen. Das Gesicht gilt dem Film nicht, wenn nicht der Totenkopf dahinter einbezogen ist : 'Danse macabre'. Zu welchem Ende ? Das wird man sehen. »

6. - Siegfried Kracauer, « Vorläufige Übersicht über ein Buch zur Ästhetik des Films » [1949], in : Id., Werke, Bd. 3, op. cit., p. 843 : «12 Der Totenkopf. Anhand einer Untersuchung von DEATH DAY, einem Kurzfilm, der aus Eisensteins mexikanischem Filmmaterial zusammengestellt wurde, wird das letzte Kapitel nicht nur das ganze Buch zusammenfassen, sondern bestimmte weitreichende Schlussfolgerungen formulieren. »

7. - Lettre de S. Kracauer à Th. W. Adorno du 16 janvier 1964, in : Theodor W. Adorno, Siegfried Kracauer, Briefwechsel 1923-1966, hrsg. von W. Schopf, Frankfurt a. M., Suhrkamp, 2008, p. 640 ; trad. française : Correspondance 1923-1966, trad. par W. Kukulies, Lormont, Le Bord de l'eau, 2018, p. 374.

8. - Ils sont maintenant réunis, dans une traduction allemande, dans S. Kracauer, Werke, Bd. 2.2, Studien zu Massenmedien und Propaganda, op. cit. 
se caractérisent par le constant souci d'articuler l'analyse de contenu et la réflexion sur les qualités distinctives des différents médias qui en sont le support ${ }^{9}$. Ils participent ainsi à l'approfondissement de la réflexion kracauerienne sur la spécificité médiale de la propagande et croisent par là ses réflexions sur l'esthétique du film. Tenter de repérer quelques-uns des points de cette rencontre en exposant ce qui la fonde conduit ipso facto sur les traces du dialogue implicite et intermittent avec Walter Benjamin.

Une remarque générale est d'emblée possible. Si l'on considère l'ensemble des écrits américains de Kracauer sur le cinéma, il apparaît qu'y persistent les deux axes, mis en évidence par Philippe Despoix, suivant lesquels s'étaient déployées dans les années 1930 leurs réflexions communes sur la propagande et le médium photo-cinématographique : la fonction de l'image fixe et la dimension spécifiquement documentaire du film ${ }^{10}$.

Dans le manuscrit de Marseille, à côté de Vertov, un autre réalisateur russe retient l'attention de Kracauer pour son usage de la prise de vue instantanée, Alexandre Dovjenko. Après l'avoir rapidement mentionné une première fois avec René Clair, il remarque : «Les stills chez Dovjenko correspondent aux 'pauses' de la musique de cirque. » Ces références sont insérées dans le contexte plus large de l'opposition, déjà présente dans les écrits sur la photographie des années 1920 et 1930, entre (la tendance vers) l'instantané et (la tendance vers) l'image : « À l'opposé des images [...] R. Clair fige le moment (dans Paris qui dort et Deux timides). Tenir compte également des stills chez Dovjenko (dans Arsenal) ${ }^{11}$. »

La référence aux stills de Dovjenko se retrouve dans sa toute première publication en anglais en 1942, Propaganda and the Nazi War Film. Les plans fixes du réalisateur russe y sont opposés aux mouvements répétitifs et incessants des caméras comme des masses dans Triumph des Willens de Leni Riefenstahl :

9. - Ces travaux sur la propagande représentent en fait des étapes décisives de son intervention au sein des sciences humaines américaines en faveur d'une approche qualitative. Voir « The Challenge of Qualitative Content Analysis », Public Opinion Quarterly, n 4, 1952-53, p. 631-642 ; trad. all. : « Für eine qualitative Inhaltsanalyse », in : S. Kracauer, Werke, Bd. 2.2, op. cit., p. 557-571.

10. - Voir son article dans ce même numéro. Pour les essais américains de Kracauer sur le cinéma, cf. Siegfried Kracauer's American Writings. Essays on Film and Popular Culture, ed. by Johannes von Moltke and Kristy Rawson, Berkeley, Los Angeles, London, University of California Press, 2012.

11. - S. Kracauer, [" Marseiller Entwurf» zu einer Theorie des Films], op. cit., p. 530 et p. 564 : « Die 'Stills' bei Dowschenko entsprechen den 'Pausen' der Zirkusmusik» et «Im Gegensatz zu den Bildern [...] arretiert René Clair den Moment (in PARIS QUI DORT und DEUX TIMIDES). Auch die Stills bei Dovschenko [sic] zu berücksichtigen (in ARSENAL). » La tendance à l'instantané, dans la mesure où elle s'oppose à l'intentionnalité de toute composition, représente un point nodal pour penser le lien entre photographie et film en même temps que pour la formulation de la tension entre tendance réaliste et tendance formatrice propres au photographe et au cinéaste. 
Pour donner corps à cette transfiguration de la réalité, Le triomphe de la volonté se laisse aller à insister sur des mouvements sans fin. [...] Dans les films du cinéaste ukrainien Dovjenko, le mouvement est parfois arrêté sur une image, qui, comme une halte, présente un fragment de la réalité sans mouvement : c'est comme si, en arrêtant toute la vie, l'essence de la réalité, son propre être, était divulguée. Ce serait impossible dans $L e$ triomphe de la volonté. Au contraire, ici le mouvement total semble avoir dévoré la substance et la vie n'existe qu'en état de transition ${ }^{12}$.

Cette étude, légèrement retravaillée, sera reprise en annexe dans From Caligari to Hitler. Il n'y a pas lieu d'entrer ici dans l'histoire de la genèse, parallèle en fait, de cet ouvrage et des travaux de Kracauer sur la propagande ${ }^{13}$. Il faut toutefois souligner que le contexte dans lequel celui-ci se confronte aussi bien avec des films dont il avait pour la plupart déjà rendu compte dans la Frankfurter Zeitung qu'avec les actualités et le cinéma documentaire sous Hitler, est double : celui de la recherche sur la propagande et la communication de masse et celui des études cinématographiques, pour lesquelles la Film Library du MoMa, avec ses collections de films et sa bibliothèque, lui offrait de nouvelles perspectives.

Dans la continuité de son étude Totalitäre Propaganda, Kracauer souligne le rôle de propagande des actualités de guerre allemandes après 1939, sans commune mesure avec les pratiques précédentes, avant d'énumérer les trois principes qui les régissent. Selon le premier, celles-ci, afin d'amplifier l'effet de réel, " devaient coller à la réalité », en n'utilisant que des « vues filmées sur le front », et ne pas recourir à la reconstitution; deuxièmement, elles devaient avoir une durée suffisante pour permettre des effets équivalents à ceux obtenus par les répétitions dans le discours ; et enfin, il était recommandé de les projeter rapidement pour que leurs effets renforcent l'impact des informations radiophoniques ${ }^{14}$. La temporalité propre de chaque médium était ainsi prise en compte, afin que leur synergie augmente leur efficacité. Dans un deuxième temps, il étudie les procédés cinématographiques utilisés dans les films de guerre, l'autoreprésentation des nazis - de Hitler jusqu'aux simples soldats de la Wehrmacht - ainsi que leur dramaturgie. Il commente les emprunts aux films russes et, par ce biais, aborde les différences qui subsistent entre la propagande soviétique et la propagande national-socialiste une thématique déjà présente dans Totalitäre Propaganda. Le schéma

12. — Siegfried Kracauer, « La propagande et le film de guerre nazi » [1942], in : Id., De Caligari à Hitler. Une histoire psychologique du cinéma allemand, trad. par C. B. Levenson, Paris, L'Age d'homme, 2009, p. 344-345.

13. - Voir la postface de Leonardo Quaresima à cet ouvrage, « Relire De Caligari à Hitler de Kracauer », ibid., p. 405-449.

14. - Cf. S. Kracauer, De Caligari à Hitler, op. cit., p. 312-314. Les actualités durent en effet presque 40 minutes parfois. 
d'« analyse structurelle » des films de propagande, présenté à la fin de l'étude, ouvre la voie à une analyse structurelle du film en général, en même temps qu'il constitue un modèle d'analyse qualitative du film de propagande et, dans une certaine mesure, du film documentaire. Erwin Panofsky ne s'y est pas trompé, quand il écrivait en août 1942, dans sa lettre de remerciement pour l'envoi du texte, que Kracauer n'a pas seulement brillamment abordé les problèmes que posaient les films de propagande nazis, mais qu'il a livré « une contribution extrêmement importante sur la structure du "film documentaire" en général », avant d'ajouter :

Je crois en effet - mais ne le répétez pas - qu'il n'existe pas de vrai « film documentaire», et que nos soi-disant Documentaries sont aussi des films de propagande, mais, Dieu soit loué, le plus souvent pour une meilleure cause, et, hélas, le plus souvent moins bien faits ${ }^{15}$.

Quand Kracauer revient dans Théorie du film sur le documentaire, le propos de Panofsky résonne dans ses analyses, qui, en même temps, renouent directement avec ses remarques antérieures sur « la fonction critique de la dimension documentaire inhérente au cinéma » ainsi que sur les liens entre avant-garde et documentaire ${ }^{16}$. Dans deux chapitres qui se succèdent, «Le film expérimental » et « Le film factuel », il aborde les différents genres appartenant à l'une ou l'autre de ces catégories exclusivement à l'aune de leur capacité à révéler l'existant matériel et de leur affinité avec l'illimité. Son approche va bien au-delà d'une simple contestation des classifications usuelles, elle revient en fait à les déconstruire ${ }^{17}$. Aussi, le traitement différencié et approfondi des divers types de films expérimentaux et documentaires se concentre sur l'explicitation des tendances ou des visées, qu'elles soient d'ordre esthétique ou intellectuel (moral, idéologique, etc.), susceptibles de contenir la puissance documentaire propre au film. Dans ce contexte apparaît à nouveau avec force l'aporétique relation de tension entre la tendance vers la constitution d'une histoire et les affinités spécifiques du film pour

15. - Lettre de Erwin Panofsky à Siegfried Kracauer du 18 août 1942, in : Siegfried Kracauer - Erwin Panofsky, Briefwechsel, 1941-1966, hrsg. von Volker Breidecker, Berlin, Akademie Verlag, 1996, p. 11 : « Ich glaube nämlich - sagen Sie das nicht weiter - dass es einen echten " Documentary Film » überhaupt nicht gibt, und dass unsere sogenannten Documentaries auch Propaganda-Filme sind, nur, Gott sei Dank, meistens für eine bessere Sache, und, leider Gottes, meistens nicht so geschickt gemacht. »

16. - Voir l'article déjà cité de Ph. Despoix dans ce même numéro, supra, p. 130.

17. - Cf. Siegfried Kracauer, Théorie du film. La rédemption de la réalité matérielle [1960], trad. par D. Blanchard et C. Orsoni, Paris, Flammarion, 2010, p. 257-308. Écartant d'emblée les questions de classification, Kracauer précise qu'il traite dans le chapitre consacré au « film factuel » des trois genres « supposés couvrir toutes les variantes concernées » : les actualités, tous types de documentaires et le film sur l'art (p. 281). 
l'illimité et le fragment qui la contredisent. Tout au long de ces deux chapitres, la question de la narration cinématographique est articulée avec la réflexion sur les affinités distinctives du médium. Les films de Robert Flaherty, " explorations du 'vaste monde' », y sont convoqués en tant qu'exemples d'œuvres qui atteignent souvent un équilibre, per se instable, entre récit et geste documentaire grâce à un mode de narration qui maintient la porosité du film envers la réalité enregistrée. Pour Kracauer, avec son « intrigue légèrement esquissée »-l'expression est de Paul Rotha - Flaherty invente une des formes d'histoire proprement cinématographiques ${ }^{18}$.

\section{L'exemple de Borinage}

Rien d'étonnant alors que Joris Ivens, déjà mentionné dans le passage sur le lien entre avant-garde et documentaire du manuscrit de Marseille, fasse partie des réalisateurs qui sont présents aussi bien dans le chapitre sur le film expérimental que dans celui sur le documentaire. Cependant, les brefs commentaires de deux de ses films, Regen/Pluie (1929) et Borinage (1933), semblent, à première vue, presque contradictoires. Film expérimental, Pluie d'Ivens bien que « saturé d'intentions formatrices » est pleinement cinématographique, dans la mesure où le cinéaste a su déployer « dans toute sa beauté le spectre d'un triste jour de pluie ${ }^{19}$ » en se laissant absorber sans réserve par ce qu'il filmait. Mais en tant qu'exploration et interprétation du « monde visible par l'image $20 »$ qui puise sa force dans le geste documentaire, il est tout autant, selon Kracauer, un documentaire. Pluie fait partie d'un type de films qui ressortit à la fois du documentaire et du film expérimental. Dans ces films, le monde environnant semble stimuler l'imagination du réalisateur, en même temps que celui-ci met sa subjectivité au service de son exploration, - autre manière d'exprimer un type d'équilibre entre tendance réaliste et tendance formatrice ${ }^{21}$. Alors que pour Borinage, que Benjamin qualifiait, avec Les trois chants pour Lénine de Vertov, d'œuvre d'art, la qualité première mise en avant par Kracauer est la retenue des réalisateurs. Affirmation a priori paradoxale si l'on se souvient que le film, tourné dans un bassin minier, se veut un documentaire engagé sur la vie des mineurs en Belgique. Non sans raison, le

18. - Il est à noter qu'une telle forme n'est jamais pure et qu'elle n'a pas à l'être. Pour Kracauer, le film est hybride, « impur ». Chez Flaherty également, on trouve des séquences construites de manière à véhiculer une thèse. Mais elles sont la plupart du temps insérées dans des passages qui ouvrent vers l'indétermination.

19. - S. Kracauer, Théorie du film, op. cit., p. 264.

20. - Ibid., p. 295.

21. - Une telle approche est qualifiée de « lecture imaginative », expression qui marque une distance avec tout geste interprétatif assignant un sens déterminé ou symbolique. 
reproche d'être un film de propagande lui a été adressé par les critiques de l'époque comme par des commentateurs plus récents. Aussi, comme pour les films de Vertov, se pose la question de ce qui dans ce film résiste à la propagande. D'autant que l'argument avancé par Kracauer en sa faveur, qui ne fait que reprendre un propos d'Ivens lui-même, pourrait sembler d'ordre moral :

Peut-être est-ce également le sujet du film qui le met à l'abri de ce genre de pulsions [esthétiques]. Joris Ivens raconte que durant le tournage de Borinage, documentaire consacré aux mineurs dans cette région minière de Belgique, Henri Storck et lui-même se rendirent compte que le sujet imposait qu'ils renoncent à tout raffinement esthétique au profit de la «simplicité » photographique. « Nous sentions que ce serait insulter les gens réduits à d'aussi dures conditions que de faire appel à un style de photographie qui ferait obstacle à la communication directe et sincère de leurs épreuves à tout spectateur. » La souffrance humaine, semble-t-il, demande à être exposée avec retenue ; ce sont les photographies dénuées de recherche artistique qui témoignent de la conscience de l'artiste 22 .

Borinage, connu aussi sous le titre Misère au Borinage, réalisé en 1933 par Henri Storck et Joris Ivens, est un film qui revient, un an plus tard, sur la grande grève des mineurs belges de 1932. Il est cependant loin d'être constitué uniquement de scènes tournées au Borinage. Ainsi que l'expose de manière programmatique et ostentatoire la première séquence du film, celui-ci est fait de matériaux hétéroclites : d'unités composées par le remontage de matériaux filmiques préexistants de diverses provenances (bandes d'actualités, scènes documentaires, etc.) et de scènes tournées sur place (Fig. 1)23.
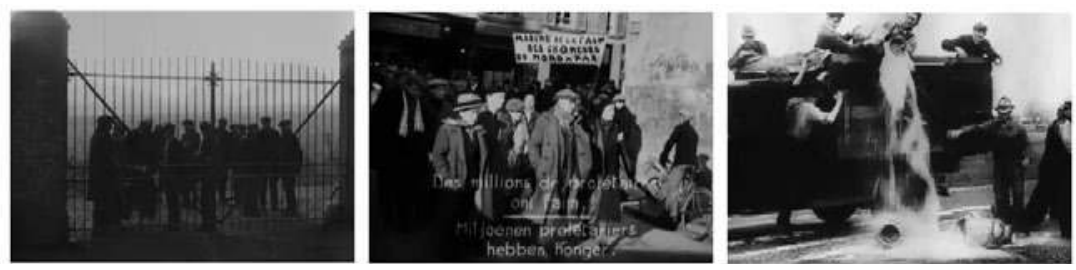

Fig. 1 : Photogrammes de Borinage (BEL, 1933)

22. - S. Kracauer, Théorie du film, op. cit., p. 294.

23. - Sur l'histoire de la genèse et du tournage du film, voir en particulier le numéro de la Revue belge du cinéma, hiver 1983 - printemps 1984. La provenance de ces matériaux est souvent connue ou identifiable. C'est le cas par exemple pour les extraits fournis par la New York and Photo League, ou ceux tournés pour le compte de RKO-Pathé lors de la sanglante répression des métallurgistes en grève en Pennsylvanie. 
Tout au long du film, ces matériaux alternent et parfois se mélangent subtilement. Les séquences tournées par Ivens et Storck sont soit des reconstitutions d'actions collectives (d'ampleurs variées), comme par exemple la résistance aux expulsions ou un rassemblement à l'initiative des syndicats, soit des reconstitutions de scènes de la vie quotidienne. Dans les deux cas, les mineurs et leurs familles jouent leur propre rôle dans leur environnement. Dans l'alternance entre séquences de remontage et séquences de reconstitution de moments de la vie des mineurs, on reconnaît deux des principales voies du documentarisme associées aux noms de ses deux tenants les plus marquants de l'époque, Vertov et Flaherty. Tous deux constituent une référence pour Ivens, comme pour Benjamin et Kracauer.

Nombreuses, les scènes de la vie quotidienne sont parfois centrées sur un micro-événement, familier et récurrent, comme le repas ou la visite du médecin, parfois elles sont simplement descriptives. Dans une séquence visant à dénoncer l'opposition entre pauvres et riches, on peut distinguer trois unités, dont la deuxième n'est qu'une simple illustration ${ }^{24}$. Dans la première unité, qui commence avec le plan arrêté de l'espace exigu d'une famille nombreuse, la caméra se met en mouvement, glisse le long des murs, frôle les objets ou les visages qu'elle rencontre. Parfois, elle s'immobilise devant un objet, comme des chaussettes trouées suspendues sur un fil, avant de reprendre son mouvement. Le troisième segment, plus bref, prolonge ce moment descriptif et se termine par le plan, fixe lui aussi, d'un mineur au chômage assis sur son lit (Fig. 2).
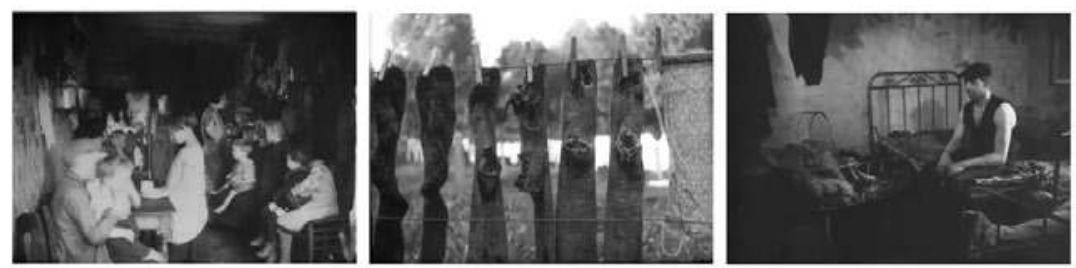

Fig. 2 : Photogrammes de Borinage (BEL, 1933)

Ce type de description n'est à l'évidence pas nécessaire pour établir l'opposition riche/pauvre, par ailleurs clairement énoncée. Si elle l'enrichit, ses effets vont bien au-delà. L'arrêt du mouvement donne au spectateur le temps de s'absorber dans l'image, lui ouvrant la possibilité d'une série d'associations et de réminiscences, que la reprise du mouvement de la caméra au ras des objets, de leur matérialité, découvrant leur texture, leurs qualités sensibles, l'entraîne à poursuivre. Le dernier plan du mineur, seul, sur son lit semble être l'image de la dépression. Il

24. - Une suite de plans, soutenue par un carton explicatif, met en contraste la richesse de l'Église avec la pauvreté des gens alentour. 
a pouvoir de convoquer, confusément, à son tour, d'autres associations et souvenirs. Plusieurs de ces plans sont extrêmement fragmentaires.

Le mouvement interrompu de la caméra semble se transmettre au spectateur en se transformant en mouvement intérieur. Comme dans les arrêts sur image de Dovjenko, ces plans fixes exercent un " pouvoir d'attraction » sur le spectateur et le captent « toujours plus intimement». La suspension du mouvement est ainsi synonyme d'une intensification de la participation du spectateur à ce qu'il voit, d'une intensification de son potentiel d'empathie. De même que l'arrêt brusque de la musique de cirque lors de l'exécution d'un numéro dangereux suscite un (type de) suspense qui fige littéralement le public avant qu'il ne soit entraîné dans une forme, souvent empathique, d'adhésion à l'action ${ }^{25}$. Dans le chapitre de Théorie du film consacré à la musique au cinéma, Kracauer renoue avec la notation de l'« Esquisse de Marseille » et développe le parallèle entre l'arrêt de la musique ou, inversement, l'irruption subite du silence dans une scène de film sonore, et l'arrêt sur image ${ }^{26}$. Parallèlement, aussi bien le moment de l'arrêt du mouvement que le malaise suscité par son prolongement provoquent chez le spectateur une prise de conscience " aiguë du fait que le mouvement constitue une partie intégrante du monde extérieur aussi bien que du film ${ }^{27} \gg$. L'image fixe assume désormais deux fonctions, que nous avons l'habitude de considérer comme antinomiques : distanciation critique et participation empathique.

Cependant, pour que ces plans fragmentaires, ces séquences radicalement descriptives et ces images arrêtées puissent assumer simultanément de telles fonctions, il faut qu'ils gardent une relative autonomie, qu'ils ne soient pas subsumés dans un discours que le montage du film pourrait construire. Dans les rares pages de Théorie du film consacrées au montage (ce fait, remarquable pourtant, n’a pas souvent été relevé), Kracauer n'énonce qu'un seul principe, un principe d'indétermination : il faut que le montage s'intéresse « aux objets représentés de façon à les faire apparaître dans toute leur indétermination suggestive ${ }^{28} \gg$. Dans Borinage, les micro-unités coexistent avec les séquences de remontage qui insèrent les événements et les situations de cette région minière belge

25. - Rappelons ici l'intérêt, et même le goût, de Kracauer pour le cirque, auquel il a consacré nombre d'articles parus dans la Frankfurter Zeitung dont le premier, "Im Zirkus », est publié en 1923. Ils sont repris dans les quatre volumes des Essays, Feuilletons, Rezensionen de Werke, Berlin, Suhrkamp, 2011.

26. - Cf. « Le suspense intensifié par le silence », in : S. Kracauer, Théorie du film, op. cit., p. 205-206.

27. — Ibid., p. 84. Dans l'« Esquisse de Marseille», dans une quasi-identification de la vie et du mouvement, Kracauer pouvait écrire : " Die leere Strasse gleicht der Pause in der Musik» (La rue vide ressemble aux pauses dans la musique), S. Kracauer, [« Marseiller Entwurf » zu einer Theorie des Films], op. cit., p. 597.

28. - S. Kracauer, Théorie du film, op. cit., p. 122. 
dans un contexte politique général, celui de la crise du capitalisme, tandis que les scènes de reconstitution de gestes de solidarité comme celles de manifestations politiques connotent idéologiquement le film. L'exposition de l'injustice sociale, de l'exploitation comme des rapports de force sociaux est articulée dans le film avec une dénonciation de l'idéologie qui, en même temps qu'elle les fonde, les recouvre et les dénie. Mais de telles révélations, soutient Kracauer, ne diffèrent guère des « messages de propagande », dans la mesure même où elles impliquent un discours idéologique. C'est d'ailleurs dans une telle perspective que s'opère dans Théorie du film une réévaluation des films de Poudovkine et d'Eisenstein, alors que certains des écrits de ce dernier constituent désormais une référence. Le potentiel spécifiquement critique du film ne relève pas de la critique de l'idéologie, il réside plutôt dans sa capacité de dévoilement des constructions idéologiques constitutives de la réalité socio-historique ${ }^{29}$.

Si Borinage n'est pas qu'un simple film de propagande, c'est parce que ces micro-unités qui relatent des événements du quotidien et décrivent les conditions matérielles de la vie des mineurs conservent une (relative) autonomie. Le montage ne soumet pas les micro-récits de la vie des mineurs et de leurs familles à des impératifs idéologiques, tout comme les aperçus de leur vie ne sont pas subsumés dans le récit de leur lutte, encore moins des luttes du prolétariat ou de la crise du capitalisme. Entre ces deux niveaux, des liens sont suggérés sans être pour autant univoquement déterminés. La discontinuité qui existe entre la micro-dimension d'un moment singulier de la journée d'une famille ou d'un individu et la macro-dimension des actions politiques, qu'elles soient locales ou pas, n'est pas effacée, l'interstice maintenu contrariant déjà toute causalité immédiate. C'est cependant la « communication directe et sincère » des conditions de leur vie, autrement dit la manière de les filmer, qui empêche toute réduction à un contenu idéologico-politique. La retenue du réalisateur devant la souffrance que revendiquait Joris Ivens n'est pas à confondre avec une attitude (soi-disant) objective, comme elle ne peut pas être entendue comme simple qualité morale. Si elle découle d'une motivation morale du réalisateur, elle n'a de sens que si elle implique une attitude face à ses matériaux induite spécifiquement par le médium cinématographique. C'est ainsi qu'elle sera mise en relation avec celle de l'historien, telle que la décrit Kracauer dans son dernier ouvrage. Il n'est pas étonnant alors que Borinage fasse partie des six films qui servent à tisser les liens entre L'Histoire et Théorie du film en vue d'établir l'analogie épistémologique entre le film et l'historiographie ${ }^{30}$.

29. - Voir le chapitre « Confrontations », in : ibid., p. 431-435.

30. - Cf. S. Kracauer, L'Histoire..., op. cit., p. 152-153. Pour un commentaire qui met l'accent sur la coexistence de différents niveaux dans Borinage selon une perspective différente, cf. Drehli Robnik, « Side by side als wirkliche Gegner. Zu 


\section{Le spectateur}

Les méditations de Kracauer aussi bien sur la narration filmique que sur l'écriture de l'histoire sont fondamentalement d'ordre épistémologique. Elles nouent dans une relation médiale à la fois l'auteur/réalisateur et le spectateur/récepteur, car la manière dont ce dernier est affecté et la facture du film sont interdépendantes, en dehors, ou plus précisément en-deçà des contenus signifiants véhiculés. Ainsi l'ultime transformation de l'image de la tête de mort en celle du bouclier offert à Persée par Athéna prend-elle toute sa signification. En effet, elle implique activement le spectateur, qui peut ainsi regarder le monstre sans en mourir. À la différence du mythe toutefois, les images terrifiantes dans le miroir de l'écran ne sont pas là en vue de rendre le spectateur capable de tuer Méduse, elles sont là pour lui permettre de vaincre la peur que suscite la perception d'un monde qui, pour reprendre une expression de Gabriel Marcel, présente, après la Deuxième Guerre mondiale, « un visage pétrifiant comme Méduse ${ }^{31} »$. G. Marcel recourt, lui aussi, immédiatement après cette remarque au mythe de Persée, pour signifier la résistance à la fascination mortifère exercée par Méduse, la résistance à la tentation d'un désespoir sans issue. Chez Kracauer comme chez Marcel, l'évocation du mythe sert à décrire une expérience subjectivement libératrice ${ }^{32}$.

L'effet de la facture du film sur sa réception s'avère constitutif de la théorisation kracauerienne du médium. Ainsi, l'interrogation inquiète des années 1930 et 1940 sur le " puissant effet de la propagande » au cinéma réapparaît dans le chapitre consacré à l'expérience du spectateur. Il s'ouvre par le constat que, devant le flux ininterrompu des plans projetés sur l'écran de la salle obscure, celui-ci se trouve "à bien des égards dans la situation de l'hypnotisé » avec une conscience « émoussée 33 ». En agissant fortement sur les sens du spectateur, le film fait surgir en lui des réactions qui influent sur la réception d'un message, sur sa disposition à l'accepter ou le refuser ${ }^{34}$.

politischen Einsätzen im Film-Denken von Siegfried Kracauers History », in : Drehli Robnik, Amalia Kerekes, Katalin Teller (Hg.), Film als Loch in der Wand. Kino und Geschichte bei Siegfried Kracauer, Wien-Berlin, Verlag Turia+Kant, 2013, p. 160-182.

31. — Gabriel Marcel, "Présence et immortalité » [1951], in : Id., Présence et immortalité. Journal métaphysique 1938-1943 et autres textes, Paris, Union générale d'éditions, 1968, p. 245.

32. - Surprenante convergence si l'on pense à tout ce qui sépare la métaphysique religieuse de Marcel de l'horizon philosophique de Kracauer. Cette distance se traduit d'ailleurs, ici aussi, par la centralité du personnage de Persée pour l'un, l'accent mis sur le reflet dans le bouclier, que tout un chacun, tout spectateur, peut regarder, chez l'autre. Rappelons que Gabriel Marcel a été un soutien très important pour Kracauer durant son exil parisien.

33. - S. Kracauer, Théorie du film, op. cit., p. 238.

34. - Dans l'« Esquisse de Marseille », le chapitre qui devait traiter du spec- 
Sur cet arrière-plan, il apparaît que l'efficacité de la propagande réside bien plus dans son pouvoir d'agir sur le public à ces niveaux préconscients, indépendamment de son contenu. Cela revient aussi à dire que les films de propagande sont d'autant plus efficaces qu'ils sont cinématographiquement bien faits. Se référant dans ce contexte explicitement à ses travaux des années 1940, Kracauer réaffirme la force des films de propagande nazis : " Ayant appris la leçon des Russes des années 1920, les cinéastes nazis, jouant sur les instincts, se révélèrent des maîtres dans l'art de mobiliser les régions crépusculaires de l'esprit ${ }^{35} \gg$. Il revient plus spécifiquement sur Sieg im Westen, déjà analysé dans Propaganda and the Nazi War Film, afin de souligner que l'efficacité des documentaires de propagande est aussi fonction de leur apparence d'authenticité, de leur caractère réaliste. Le propos évoque irrésistiblement les dernières notes de Benjamin sur les convergences entre ses analyses et celles de Kracauer, quand il constatait combien l'accentuation de l'effet de réel devait amener à détruire "l'image d'une réalité totale 36 ». Mais il attire en même temps l'attention sur le fait, central pour la conception kracauerienne, que la dimension réaliste du film est reliée au « réalisme propre à la caméra » et donc indépendante de tout réalisme de la représentation comme des genres dits réalistes. Autrement dit, le potentiel documentaire spécifiquement filmique qui lui est associé n'est pas le propre du genre documentaire ${ }^{37}$.

Or, un état de conscience atténué, tel que celui du spectateur de cinéma, incite au rêve. En préambule aux développements sur le caractère onirique du film, Kracauer, afin de prévenir quelques confusions probables, consacre un rapide commentaire aux « rêves » hollywoodiens. "Rêves éveillés », ceux-ci se manifestent essentiellement dans l'intrigue et « bien souvent, note-t-il, c'est comme un élément extrinsèque qu'ils s'imposent au médium ${ }^{38} »$. Généralement révélateurs de besoins et de courants sociaux, ils n'ont toutefois qu'un mince intérêt esthétique. Sans expliciter plus avant le sens de cette affirmation, Kracauer, dans un raccourci saisissant, énonce d'emblée sa conception : « Ce qui compte ici, ce ne sont pas les fonctions et les implications sociales du médium en tant que véhicule d'un divertissement de masse ; la question est de savoir

tateur s'intitule « Mit Haut und Haaren » et comporte un passage sur le film comme instrument de propagande, cf. S. Kracauer, [« Marseiller Entwurf» zu einer Theorie des Films], op. cit., p. 559, 575.

35. - S. Kracauer, Théorie du film, op. cit., p. 240.

36. - Voir l'article de Ph. Despoix dans ce numéro, supra, p. 131.

37. - Une telle conception s'est élaborée au fil du temps et des écrits de Kracauer. Aussi, les lire à rebours, en partant de Théorie du film, permet d'éviter quelques malentendus, en particulier dans le cas de From Caligari to Hitler.

38. - S. Kracauer, Théorie du film, op. cit., p. 244. 
si le film en tant que film comporte des éléments de caractère onirique qui contribuent à plonger le spectateur dans le rêve ${ }^{39}$. "

En quelques pages denses, Kracauer présente l'expérience du spectateur au cinéma comme un type particulier d'activité onirique se déployant dans deux directions opposées. À condition qu'un film soit suffisamment poreux, " troué », pour qu'il laisse des sensations et des significations indéterminées, obscures, flottantes, se détacher de la matérialité même des choses, le spectateur est libre de se perdre dans les plans et leur succession. Dans un mouvement littéralement d'aliénation, il s'identifie en quelque sorte à tous les objets animés et inanimés qui irrésistiblement l'attirent. Mais, en même temps, les images filmiques l'affectent de manière à provoquer un mouvement inverse d'immersion en soi qui fait remonter des souvenirs involontaires et naître des émotions et sensations fugitives. En décrivant cette expérience dans des termes proustiens - une perception aliénée et une expérience sensible déclenchant les souvenirs et l'imagination créatrice -, Kracauer établit ici une chaîne d'homologie reliant cinéaste/producteur et spectateur/récepteur. L'abolition de la frontière entre producteur et récepteur est désormais pensée indépendamment du type d'individus représentés à l'écran ${ }^{40}$; potentiellement inhérente au film conçu en adéquation avec les qualités propres au médium, elle est en fait indépendante de tout contenu prédéfini.

Dans les années 1920 et 1930, la question de la tension entre l'intention, y compris propagandiste, d'un film et ses effets sur le public préoccupait Kracauer comme Benjamin. Kracauer poursuit cette réflexion aux États-Unis, tout en lui donnant une nouvelle impulsion. En effet, l'élucidation dans Théorie du film des affinités intrinsèques du médium photo-cinématographique débouche sur une compréhension approfondie de l'expérience cinématographique en même temps que du sujet de celleci. Kracauer voyait dans la tendance vers l'abstraction, indépendante de toute idéologie, la caractéristique fondamentale de l'époque. Ses conséquences étaient d'autant plus délétères qu'elle était généralisée. L'expérience cinématographique, « appréhension esthétique » du monde en ce qu'elle permet de saisir les phénomènes matériels dans leur concrétude, allait à l'encontre de celle-ci. À travers la figure du spectateur de cinéma, Kracauer proposait l'esquisse d'un sujet qui était d'autant plus capable de résister à l'emprise de la propagande qu'ayant rétabli un lien concret avec le monde matériel environnant, il était à même de s'opposer à l'abstraction inhérente aux discours idéologiques. L'épilogue du livre, intitulé « Le film à notre époque », entrelace considérations théoriques

39. - Ibid.

40. - Les considérations sur l'acteur, que les films néoréalistes italiens en particulier ont contribué à enrichir, intègrent les considérations sur les individus anonymes à l'écran. 
sur le cinéma et réflexions concrètes quant aux possibilités d'existence d'un tel sujet dans les conditions historiques de l'après-deuxième guerre mondiale, d'après la Shoah. Question dont l'urgence critique s'imposait alors, et que Kracauer poursuivra dans son ultime ouvrage, resté inachevé, History. The Last Things Before the Last. Une telle problématique conserve, selon nous, dans les conditions médiales d'aujourd'hui plus que jamais pertinence et actualité. 
